\title{
Ueber die intraperitoneale Stielbehandlung bei der Myomektomie.
}

\author{
Von \\ P. Zweifel in Leipzig. \\ (Mit 5 Abbildungen und \& Curventafeln.)
}

Während Ovariotomie und Myomektomie an sich zwei nahe verwandte Operationen sind und, so lange die extraperitoneale Stielbehandlung auch bei der Ovariotomie die Oberhand hatte, im Wesentlichen ganz gleich ausgeführt wurden, gehen die Verfahren, seitdem alle Operateure bei der Ovariotomie zur intraperitonealen Stielbehandlung zurückgekehrt sind, weit auseinander. Die Ovariotomie ist einfacher und erfolgreicher geworden, für die Myomektomie überbieten die Operateure einander an Kühnheit und Schneidigkeit im Lösen des gordischen Knotens.

„Nicht die intra-, nicht die extraperitoneale Methode der Stielbehandlung ist die Operation der Zukunft, sondern die Totalexstirpation des myomatösen Uterus, " lautete es in Berlin und hallt es jetzt von mehreren Seiten wieder. Schon beginnen die Prioritätsansprüche sich zu regen, zum Zeichen, wie man dem kühnsten Verfahren schon eine goldene Zukunft verspricht.

Es ist recht, dass diese Uebertreibung in die Welt kommt, um so eher und rascher wird der Rückschlag folgen.

Es ist wesentlich, die Vortheile der intraperitonealen Stielbehandlung der Ovariotomie sich klar zu machen, um an die jetzigen Methoden der Myomektomie den richtigen Maassstab anzulegen.

Ein Ovarialstiel ist in der Regel so lang, dass er ohne Zerrung auf der Bauchwand befestigt werden kann. Der Stiel der Myome ist dagegen meistentheils der Gebärmutterhals. Seine natürliche Lage ist so tief im Becken, dass die Befestigung der Cervix uteri auf den Bauchdecken niemals ohne heftige Zerrung, 
ohne eine gewaltthätige Ortsveränderung möglich ist, Je nachdem die Theile, d. h. die Bauchdecken, eindrückbar und der Uterus leicht beweglich sind, wird dies mehr oder weniger Schmerzen und Qual machen. Nur wo gestielte, dem Fundus aufsitzende Myome zu entfernen sind, fällt die Zerrung ganz weg.

Nun wird der nach aussen geleitete Stiel abgeschnürt. Je fester, je vollkommener die Abschnürung stattfindet, um so kürzer ist der Schmerz. Je grösser die Massenligatur ist, um so qualvoller und länger ist der ,Todeskampf der absterbenden Nerven“. Die Ovarialstiele sind leicht so fest zu schnüren, dass nach wenig Stunden alle Nerven an der Schnürungsstelle taub und todt sind. Bei dem dicken Gebärmautterhalse mit seinem derben Gewebe braucht es bei ein bis zwei Massenligaturen weit länger. Der Schmerz war in allen Fällen, die ich bei extraperitonealer Stielbehandlung beobachtete, $2 \mathrm{Mal} 24$ Stunden nach der Operation so qualvoll, dass die Kranken laut jammernd und schweisstriefend dalagen.

Es sind zwei Momente, welche den heftigen Schmerz bedingen: die Zerrung der Gebärmutter und das relativ langsame Abschnüren, beziehungsweise Absterben des Gewebes. Die eine dieser Ursachen fällt bei der intraperitonealen Stielbehandlung ganz weg, die andere - der Abschnürungsschmerz - ist bei Abbindung kleinerer Massen auf ein Minimum wie bei der Ovariotomie zu beschränken.

Das Bild der Genesenden nach der intraperitonealen Stielbehandlung ist grundverschieden von dem bei der extraperitonealen.

Ein zweiter Nachtheil der Aussenbehandlung des Schnürstückes ist dessen Mortification. So oft die Luftkeime nicht vollkommen abgeschlossen werden, entsteht eine ekelhafte, blutigjauchige Absonderung aus dem Schnürstumpfe. Natürlich hängt dessen Menge wesentlich von der Grösse des Schnürstïckes ab. Selbst bei Chlorzinkeintrocknung, welche von Hegar eingeführt wurde, ist diese Absonderung nicht ganz zu vermeiden, aber einzuschränken und aseptisch zu halten.

Diese Absonderung bildet in allen Fällen eine Gefahr für nachträgliche Infection, wenn der Geschwulststumpf nicht sorgfältig von der Bauchhöhle abgeschlossen wird. Es ist nicht zu leugnen, und die vielen günstigen Erfahrungen der extraperito- 
nealen Behandlung beweisen es, dass bei langem Stiele und sorgfältiger Umsäumung diese Gefahr vermieden werden kann.

Immer aber erfordert der aussen festgehaltene Myomstiel, bezw. Uterusstumpf eine lange, sorgfältige Nachbehandlung. Erst in der dritten Woche pflegt die abgeschnürte Cervix uteri sich abzulösen; dann muss die zurückbleibende Granulationsfläche sich noch überhäuten, ehe die Heilung vollendet ist.

Drittens bleibt an der Stelle des herausgeleiteten Myomstumpfes stets eine schwache Stelle der Bauchwand, an der im späteren Verlaufe in der Regel eine Bauchhernie entsteht.

Einer der wichtigsten Gegengründe ist endlich, dass in allen Fällen, wo sich das Myom unter die Serosa der Ligamenta lata entwickelt hat, die extraperitoneale Stielbehandlung gar nicht oder doch erst nach der Ausschälung aus der Serosa möglich ist und nun die so zurückzulassende, am Stumpfe anliegende Wundhöhle die Gefahr der nachträglichen Infection bedeutend vergrössert.

Warum zaudern die Fachgenossen, zur intraperitonealen Stielbehandlung überzugehen, wie es alle bei der Kystomektomie gethan haben?

Die Gründe sind die früheren schlechten Erfahrungen: Nachblutungen aus dem versenkten Stumpfe und daraus folgende peritoneale Infectionen.

Wir brauchen aber nur die Lehren der Ovariotomie auf die Myomektomie zu übertragen, um die Einwendungen gegen die Versenkung der Myom- beziehungsweise der Uterusstümpfe gegenstandslos zu machen.

Man schnürt den Ovariokystomstiel mit voller Kraft ab, setzt auch hierbei ein kleineres oder grösseres Stück Gewebe vollkommen ausser Ernährung und versenkt es im Vertrauen auf die Asepsis, weil die Erfahrung gelehrt hat, dass es dann ohne Gefährdung resorbirt wird.

Warum will man nicht genau ebenso mit dem Reste der Cervix uteri oder dem Stumpfe des Myoms verfahren? Man hatte ihn im Anfange, als diese Operationen aufkamen, in zwei Partien ligirt und versenkt, dies zeigte sich als ungenügend. Als K. Schröder vor zwölf Jahren die Myomotomie und besonders die Stielversenkung wieder aufnahm, machte er dasselbe Verfahren und wohl auch die gleichen Erfahrungen noch einmal. Er liess die doppelte Unterbindung wieder fallen und ging zu der von ihm ausgebildeten Methode der Etagennähte über. 
Was die intraperitoneale Methode gegenüber der Aussenbehandlung voraus hat, besitzt die Schröder'sche Etagennaht in vollem Umfange. Aber keine Naht giebt dieselbe Garantie für absolute Blutstillung, wie Ligaturen. Wo man Sicherheit gegen Blutungen haben will, wendet man in der gesammten Chirurgie nicht Nähte, sondern Ligaturen an.

Um auch mit Unterbindungen volle Compression der Gefässe zu erzielen, müssen nur die zu unterbindenden Theile nicht zu gross genommen werden. Je starrer ein Gewebe ist, um so mehr verdient dieser Grundsatz Beachtung.

Die volle Sicherheit gegen Blutungen aus dem Stumpfe lässt sich leicht erreichen durch ein Verfahren, welches ich als fortlaufende Partienligatur (Theilunterbindung) oder Ligatura partialis continua bezeichnet habe. Es gestattet das Verfahren, grössere Stiele in beliebig vielen einzelnen Theilen rasch zu durchstechen und abzuschnüren, um dann nach Schluss der Ligaturen vollkommen sicher zu sein, dass alles Gewebe abgebunden ist.

Die fortlaufende Partienligatur im Allgemeinen und deren besondere Vorzüglichkeit für die Myomektomie habe ich schon einmal veröffentlicht - , ich kann mich deshalb kürzer fassen und auf die frühere Publication ${ }^{1}$ ) verweisen.

Sie wird ausgeführt mit einer langen gestielten und gekrümmten Nadel, welche die Eigenthümlichkeit besitzt, dass ihr Oehr vom Handgriffe der Nadel aus geöffnet und geschlossen werden kann. Dadurch ermöglicht sie, in einem Stichcanale einen Faden hinauszubringen und einen zweiten auf demselben Wege zurückzunehmen. Es ist einleuchtend, dass auf diese Weise bequem grössere Gewebsbrücken in einzelnen Theilunterbindungen abzustechen und in den einzelnen Partien fest zu ligiren sind.

Die Abbildung Fig. 1 sollte weitere Erklärungen ersparen. Zu den Ovarialstielen und den Ligamenta lata bei den Myomohysterektomien benutze ich die von mir modificirte stumpfe Schiebernadel, für den Uterusstumpf ist die scharfe Bruns'sche Schiebernadel unerlässlich. Das Tauschen der zwei Instrumente kann bei jedem Faden geschehen.

1) Centralblatt für Gynäkologie 1884, S. 405 und: Die Stielbehandlung bei der Myomektomie. Stuttgart 1888. S. 43 u. ff.

Archiv f. Gynäkologie. Bd, XLI, Hft. 1 u. 2. 
Es beginnt die Myomektomie mit dem Herauswälzen der Geschwulst und dem Abbinden der beiden Ligamenta lata. Mit zwei, höchstens drei Theilunterbindungen lassen sich dieselben hinter und unter dem Ovarium weg bis an die Kante des Uterus heran durchstechen und abbinden. Sollte es nöthig sein, daran zu erinnern, dass durch das breite Mutterband grosse, dünnwandige Venen ziehen, die man zu vermeiden habe? Natürlich blutet es aus den Stichcanälen, wenn man solche Venen mitten durchsticht, woran auch die nachherige Schnürung der Fäden, wenn sie nicht in Kettenstich durchgezogen werden, nichts ändern könnte.

Die Durchstichstellen lassen sich durch Befühlen und Sichtbarmachen leicht finden, und nur davor möchte ich warnen, die-

Fig. 1.

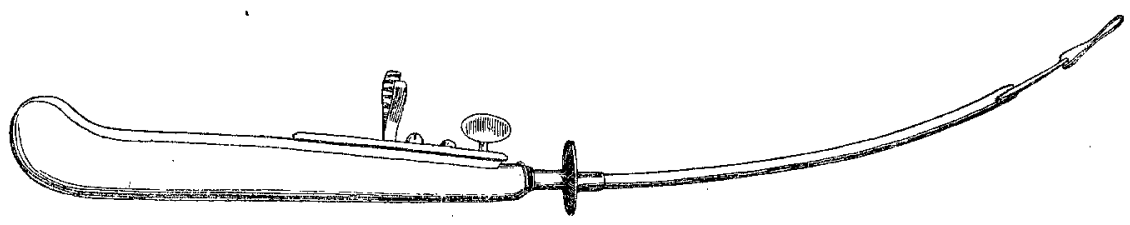

Die stumpfe Schiebernadel.

jenige Ligatur, welche nach der Excision des Corpus uteri die letzte Falte des Ligamentum gegen die Kante des Uterus schnüren soll, zu nahe an den Uterus heran legen zu wollen, sonst trifft man sicher auf Gefässe und erlebt eine zwar nicht gefährliche, aber ganz entbehrliche und vermeidbare Blutung.

Verfolgen wir noch einmal das Anlegen der Theilunterbindungen, so beginne ich am Ligamentum infundibulo-pelvicum, führe die stumpfe Schiebernadel hinter einer etwa fingerbreiten Partie an gefässloser Stelle durch, lasse den ersten Faden aus dem Oehr der Nadel ziehen und den zweiten hineinlegen. Diesen zweiten Faden ziehe ich zurück und führe ihn wieder fingerbreit weiter medianwärts durch eine gefässlose Stelle des Ligamentum latum, doch nicht zu nahe am Uterusrande durch, weil dort immer stark blutende Gefässe liegen. Der zweite Faden wird aus dem Oehr genommen und der dritte eingelegt. Nr. 1 und 2 werden geknotet und kurz abgeschnitten, bei Nr. 3 werden an beiden Enden Köberle'sche Klemmzangen angelegt, um diese Fäden vor Verwicklung $\mathrm{zu}$ bewahren. 
Es folgt auf das Knoten der beiden Ligamenta lata das Durchschneiden der letzteren. Damit nicht das Corpus uteri sein Blut in die Bauchhöhle ergiessen könne, wird über die Stümpfe der Tube und des Ligamentum latum jederseits eine Billroth' sche Klemmzange mit Sperrhaken angelegt.

Fig. 2.

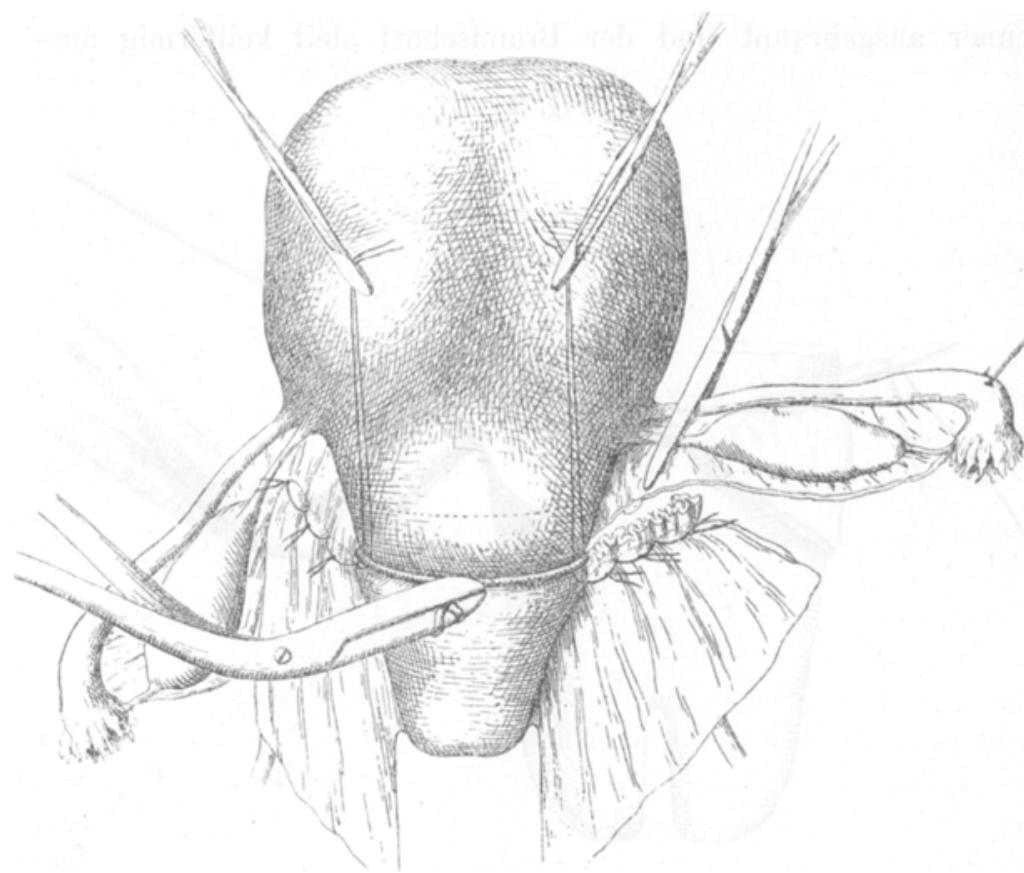

Schematische Zeichnung über das Verfahren bei der Abbindung der Ligamenta lata.

Diese Klemmzangen und die zwei nicht unterbundenen Fäden werden hoch gehalten und um den Gebärmutterhals ein elastischer Schlauch gelegt, fest angezogen und mit einer besonderen Schlauchklammer gespannt erhalten. Die Klemmzangen würden beim weiteren Operiren hindern and werden deshalb in diesem Momente abgenommen.

Unter dem Schutze des provisorisch ligirenden Schlauches folgt die Ausschneidung der Gebärmutter. Sowohl hinten als vorn soll man mit dem Messer das Gebiet des abstreifbaren Peritoneum aufsuchen, erst dieses allein durchschneiden, mit 
einem Fingernagel zurückstreifen und gleichsam eine Manchette bilden, wie dies bei den Circuläramputationen üblich ist. Vorn gelingt dies leicht, weil das Peritoneum viel weiter hinauf in lockerer Verbindung mit der Muskulatur steht. Hinten muss man bisweilen darauf verzichten und höher durchschneiden.

Nachdem die Geschwulst, bezw. das Corpus uteri ausgeschnitten ist, wird zuerst die Cervix uteri mit dem Thermokauter-Flachbrenner ausgebrannt und der Brandschorf steil keilförmig aus-

Fig. 3.

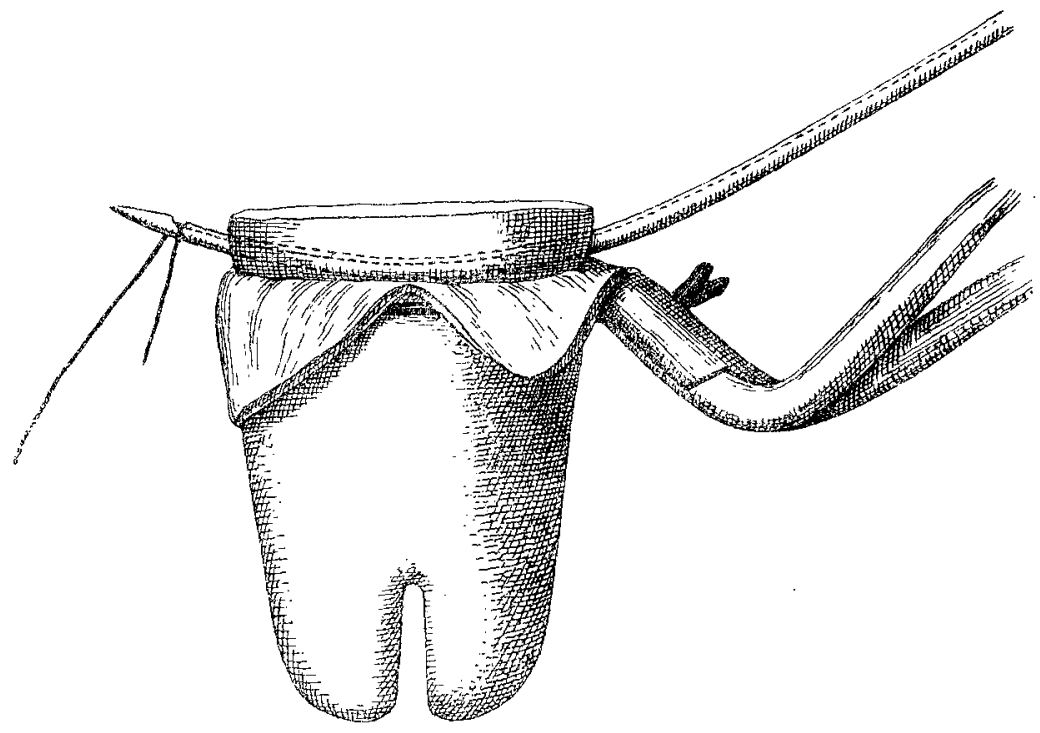

Das Abpräpariren der Peritoneallappen und das Durchstossen der Nadeln durch den Uterusstumpf, von der Seite gesehen, darstellend.

geschnitten, um allfällige Keime des Cervicalcanales zu zerstören, doch aber auch den Brandschorf nicht zurückzulassen.

Erst nach dieser Ausschneidung beginnt die Vollendung der Partienligatur durch das Collum oder Corpus uteri. Es wird das eine Ende einer nicht geknoteten Catgutligatur Nr. 3 in die scharfe, leicht gebogene Bruns'sche Schiebernadel gelegt und die Kante des Uterus unter Mitfassen des Peritoneum von vorn nach hinten durchstochen. Hinten kommt das Fadenende heraus, ein neuer Catgutfaden in das Oehr, doch wird diesmal hinten das Peritoneum nicht mehr mitgefasst, die Nadel also erst hinter die Serosa zurückgezogen und dann die Ligatur eingelegt. Auch vorn 
wird der Faden unter Vermeidung des Peritoneum aus der Nadel gezogen.

Die Fortsetzung der Theilunterbindungen kann ich kurz beschreiben. Das Princip besteht darin, dass an den Kanten des Uterus die Ligamenta unter Mitfassen der Serosae durchstochen werden, um der Unterbindung der Arteriae uterinae sicher zu sein, dagegen in der Mitte des Collum das Peritoneum nicht zu unterbinden, um dasselbe für die Ernährung des abgebundenen Stückes Gebärmutter zu erhalten (s. Fig. 3).

Fig. 4.

Fig. 5.
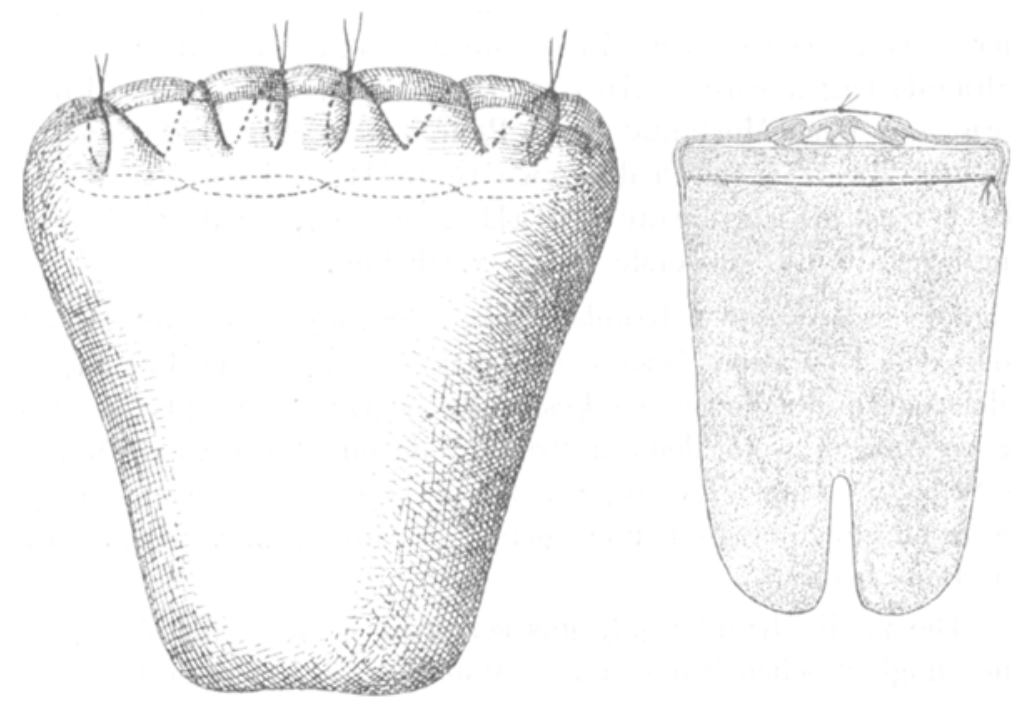

Schematische Zeichnungen. Fig. 4. Uterusstumpf mit den versenisten Theilunterbindungen von vorn, Fig. 5, die Decknaht von der Seite her gesehen.

Nach Vollendung der Partienligatur schliesst die Uebernähung des Stumpfes mit dem gelockerten Peritoneum, welches, wie der Strumpf über ein Bein, über den schmalen unterbundenen Gewebskegel gestïlpt wird, die Operation ab (s. Fig. 4). Ob diese Vereinigung der Peritonealränder mit fortlaufender oder unterbrochener Naht ausgeführt werde, macht keinen Unterschied aus.

Damit ist die Stielbehandlung vollendet, es wird die Bauchhöhle genau gereinigt und in üblicher Weise geschlossen.

Gewöhnlich macht es im Anfange der serösen Decknaht den Eindruck, als ob die Ränder nicht über dem Stumpfe zu vereinigen wären. Doch wie die ersten Decknähte liegen, wird die 
Vernähung immer leichter. Natürlich werden die eigentlichen Stumpf ligaturen schrittweise kurz geschnitten, sowie derselbe unter dem umhüllenden Peritoneum verschwindet. Nach vollendeter Operation sieht man nichts weiter als die seröse Decknaht (s. Fig. 5).

Nach dieser Methode habe ich bis jetzt hier 50. Myomektomien ausgeführt, dazu kommen noch 3 früher nach derselben Methode Operirte, so dass im Ganzen über $53 \mathrm{zu}$ berichten ist. 2 fallen von der Rechnung aus, weil sie an Krankheiten ganz anderer Art längere Zeit nach der Operation gestorben sind. Todesfälle, die nur irgendwie in einem wenn auch nur ganzentfernten Zusammenhange mit der Stielbehandlung stehen, sind unter 51 Fällen 2 vorgekommen, was einer Mortalität von 4 Proc. entspricht. Dabei sind die letzten $27 \mathrm{Fälle}$, also die zweite Hälfte, in einer Reihe glatt und ohne $Z$ wischenfall genesen, so dass sich die Unglücksfälle auf die erste Hälfte vertheilen.

Die eine dieser 2 Kranken war sehr ausgeblutet und elend; nach der Operation bekam sie den Anfang einer Pneumonie, welche schon wieder in der Besseruug .begriffen war, als plötzlich Erscheinungen von Ileus auftraten, denen die Kranke binnen 24 Stunden erlag. Es zeigte sich, dass eine Dünndarmschlinge am Stiele angewachsen und geknickt worden war. Dies hatte den Ileus bedingt.

Die zweite Kranke galt uns schon als geheilt, als sie infolge eines unglücklichen Zufalles vier Monate nach der Operation verstarb. Die Kranke hatte in der Convalescenz eine brettharte Infiltration der rechten Bauchwand bekommen, die sich lange nicht erweichen und nicht resorbiren wollte. Ohne dass nennenswerthe Fiebergrade da waren, magerte die Fran mehr und mehr ab. Der Verdacht einer tuberkulösen Infiltration war um so mehr zu rechtfertigen, als in der einen exstirpirten Tube Tuberkel und auf den mikroskopischen Durchschnitten Riesenzellen gefunden worden waren.

Es begann die Infiltration zu schmelzen und entleerte sich ein dünner Eiter. Mehrmals war die Haut vollkommen zugeheilt, um wieder aufzubrechen. Um der langweiligen Eiterung ein Ende zu machen, wurde die Haut gespalten, die Abscesshöhle ausgespült und dabei beobachtet, dass sich auch Eiter aus dem Cervicalcanal entleere. Es wurde ein Drainrohr von der Bauchwand 
durch den Uterusstumpf bis in die Vagina gelegt und die Höhle oben mit Jodoformgaze ausgestopft. Ohne unseren Befehl und ohne unser Wissen wurde statt abgekochten Wassers zum Ausspülen der Höhle eine Sublimatlösung von $0,5-1 \%$ genommen. Am Nachmittage desselben Tages collabirte die Kranke, ohne Fieber wurde der Puls kleiner und frequenter. Eine Blutung war ausgeschlossen, eine acute Vergiftung das Wahrscheinlichste. Trotz aller Stimulantien und einer Transfusion zur Hebung der geschwächten Herzkraft starb die Kranke in der darauffolgenden Nacht.

Der Secant (Herr Dr. Schmorl) konnte nirgends eine Communication der Abscesshöhle mit der Bauchhöhle nachweisen. Doch war in der Bauchhöhle ein dünnflüssiger, gelblich gefärbter Erguss, die Darmschlingen im Allgemeinen unverwachsen, aber tief kupferroth, und wenige ganz frische Fibrinverklebungen von einzelnen Schlingen mit dem Peritoneum parietale. Die Ureteren, die Blase unversehrt. Doch war der rechte Harnleiter, welcher am Rande der Abscesshöhle verlief, stark verdickt.

Links vom Uterusstumpfe, welcher ganz gut aussah, keine Infiltration, keinen Tropfen Eiter enthielt, an der Basis der Tube, entleerte sich beim Einschneiden ein frischer Abscess, etwa einen Esslöffel Eiter enthaltend, und daneben, aus der linken Tube, ein alter, käsiger Eiter, welcher ganz die Farbe und Consistenz der käsigen Abscesse der Kaninchen besass. Die Innenfläche dieser linken Tube war ebenfalls mit Tuberkeln besetzt, wie es die rechte gewesen. Die übrigen Organe waren-frei von Tuberkulose.

Wenn es auch nicht bestimmt zu entscheiden ist, dass eine acuteste Sublimatrergiftung zum Tode führte, so ist dies doch wahrscheinlich. Leider war versäumt worden, den Inhalt der Bauchhöhle aufzufangen, um ihn auf Quecksilber zu untersuchen.

Das könnte nun freilich nicht der Grund sein, diesen Fall als irgendwie in einem entfernten Zusammenhange mit der Stielbehandlung zu bezeichnen. Aber es wäre möglich, dass die Abscessbildung damals ihren Ausgang vom Stiele genommen hätte; ich kann diesen Einwand nicht unbedingt leugnen, obschon manche Gründe für eine Senkung des Abscesses von der Bauchwand sprechen, besonders der, dass im Anfange innen keine Resistenz gefühlt wurde, als aussen die Bauchdecken bretthart infiltrirt waren. Doch wäre auch dann nur durch eine seltsame, unglück- 
selige Verkettung von Umständen der Tod durch die Stielbehandlung herbeigeführt worden.

Die 2 Fälle, welche ich ausser Rechnung lasse, waren eine Kranke, welche septisch schwer krank, nachdem sie schon vorher einen Schüttelfrost gebabt hatte, zur Operation kam und vier Wochen nachher, nach 13 Schüttelfrösten, einem zerfallenden, wandständigen Thrombus der Vena cava inferior erlag. Diese kam zu spät zur Operation. Dass eine schon begonnene Phlebitis mit Schüttelfrösten vor der Operation nicht das Conto irgend einer Stielbehandlung belasten kann, braucht keine Begründung.

Die zweite Kranke hatte eine äusserst glatte Genesung begonnen, in den ersten sieben Tagen bei zweistündlichen Messungen nicht ein einziges Mal die Temperatur von $37,6^{\circ} \mathrm{C}$. erreicht, nie die Pulszahl von 88 ïberschritten, nie Erbrechen gehabt, keine Klagen geäussert.

Es begannen jedoch nach Entfernung der Bauchnähte, was regelmässig am achten Tage geschieht, alle Stichcanäle stark zu eitern. Die Frau war sehr fett, pastös und blutarm. Am 13. Tage nach der Operation wurde auf die abscedirenden Bauchdecken etwas gedrückt, um den Eiter zu rascherem Abflusse zu bringen; die Kranke seufzte ein wenig, begann jedoch $1^{1} / 2$ Stunden nachher eigenthümlich apathisch zu werden. $4 \mathrm{Uhr}$ Nachmittags wird die Athmung beschleunigt, zwei Mal geringes Erbrechen und Abends 11 Uhr 20 Minuten tritt der Tod ein.

Bei der Section fand sich Eiter, welcher aus den Stichcanälen der Haut sich nach innen in die Bauchhöhle ergossen hatte, - oder sagen wir ehrlich und offen, durch den Druck auf die eiternde Bauchwand nach innen entleert worden war!

Es ist dieser letzte Fall ein äusserst seltenes Missgeschick, das die Vorschrift einschärft, niemals Eiterungen der Bauchwand durch Druck entleeren zu wollen!

Niemand wird mir aber conditionale Statistik vorwerfen wollen, wenn ich diesen Fall nicht unter die Zahl der Myomektomien aufnehmen und danach die Mortalität berechne, wo es auf die Beurtheilung der Stielbehandlung ankommt. In die Gefahrenklasse der Laparatomien gehört dieser Unfall selbstverständlich, nicht aber speciell zu derjenigen der Myomektomien.

Im Uebrigen hat die Methode doch ihre Schuldigkeit so gut erfüllt, als nur zu wünschen ist, auch als die extraperitoneale. Ls braucht durchaus nicht bewiesen zu werden, dass die Mortalität bei der intraperitonealen Stielbehandlung geringer sei, als bei der extraperitonealen. Wenn die erstere nur gleich günstige Ziffern erreicht, so ist sie um der anderen Vortheile willen, besonders aber, weil sie leistungsfähiger ist, der extraperitonealen 
überlegen. $3 \mathrm{Mal}$ unter den 50 Fällen waren zersetzte Myome mit Stielversenkung behandelt, und während um der Verjauchung willen bis zur Operation beide Male hohes Fieber herrschte, fiel es unmittelbar nachher ab und erfolgte ungestörte Genesung.

Auch in allen übrigen Fällen war der Heilungsverlauf ganz wie nach Kystomektomieen im Allgemeinen, noch besser als nach den Salpingektomien.

Um den Fachgenossen so viel Einblick in den Verlauf zu bieten, als durch Worte und ohne Augenschein möglich ist, habe ich über alle Fälle in der Weise eine Durchschnittscurve der Körperwärme berechnet, dass die sämmtlichen, zu den gleichen Tagesstunden gefundenen Temperaturen addirt und daraus das Mittel berechnet wurde.

Curve I. Diese Curve erreicht nicht einmal die obere Grenze des Normalen, 37,6 ${ }^{\circ}$. Es fand sich eine einzige Kranke mit dabei, welche die ersten drei Tage wiederholt über $38,0^{\circ} \mathrm{C}$. maass.

Zum Vergleiche mit dieser Curve I will ich gleich eine Curve II anreihen, die das Mittel darstellt aus zwei Einzelcurven von Kranken, deren Stiel nicht fortlaufend unterbunden, sondern mit Matratzennähten behandelt wurde. Um mich genauer auszudrücken, so wurde in diesen 2 Fällen versuchsweise nach $\mathbf{A b}$ bindung der Ligamenta lata und der zwei Uteruskanten zwar ebenfalls der Cervicalcanal verschorft und steil trichterförmig ausgeschnitten, aber es wurde die Mitte des Stumpfes nicht mehr durch Unterbindung aus der Frnährung ausgeschaltet, sondern es wurden blos die einzelnen Gefässe und der Cervicalcanal durch Matratzennähte geschlossen und darüber die peritonea Decknaht wie sonst geschlossen. Mit der Blutstillung hörte ich nicht früher auf, als bis nirgends mehr ein Tröpfchen durchsickerte.

Diese Curve II zeigt recht bedeutende Temperaturerhöhungen. Da im Uebrigen das Allgemeinbefinden ungetrübt, kein Erbrechen, keine Schmerzhaftigkeit des Abdomen, keine belegte, borkige Zunge u. s. w. vorhanden war, ist dieses Fieber als sogenanntes "Resorptionsfieber" zu bezeichnen.

Was bedeutet dies, was soll resorbirt werden? Ich antworte: ,das nachträglich zwischen den Nähten in die Bauchhöhle geschwitzte Blut".

Eine einzige Operirte hatte bei Anwendung der Partienligatur mehrere Tage Fieber über $38,0^{\circ} \mathrm{C}$. Auch dort nehme ich eine Unvollkommenheit der Blutstillung an. 
$74 \quad$ Zweifel, Ueber die intraperitoneale Stielbehandlung
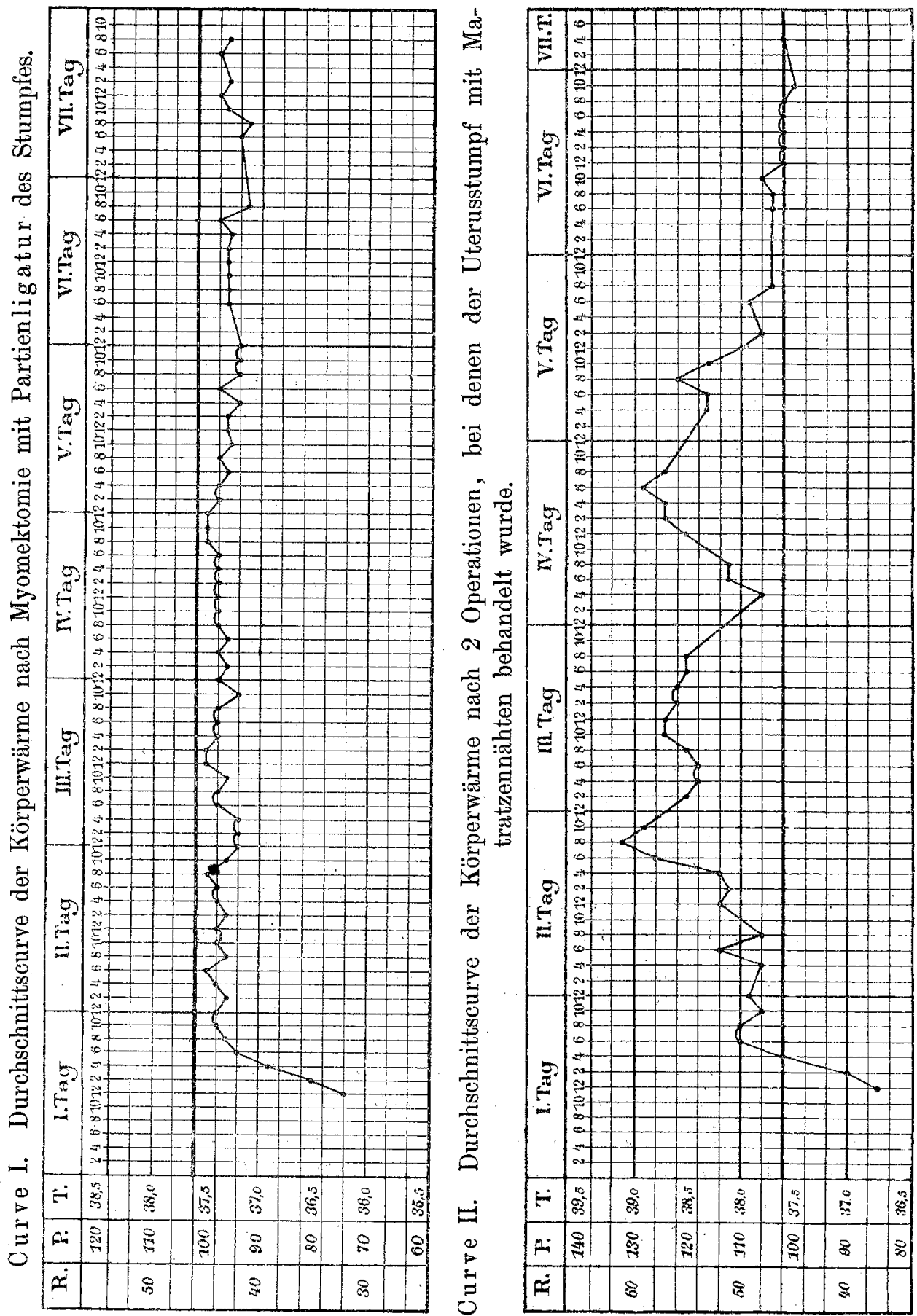
Beweisen kann ich dies zwar von keinem der 3 Fälle, aber schliessen aus einer genügenden Zahl anderer Erfahrungen.

Ich kehrte nach diesen 2 Versuchen zur vollkommneren Blutsicherung mittels der Partienligaturen zurück.

Die obigen Angaben vermögenein Bild zu geben von der Leistungsfähigkeit dieser Art von Stielbehandlung, ein umfassendes, eingehendes, vollkommen objectives, und dies ist meine Antwort auf die unfreundliche Kritik, welche dieses Verfahren auf der Versammlung der deutschen Gynäkologen in Halle 1888 gefunden hat.

Ich kann an dieser Stelle das Gefühl nicht unterdrücken, dass damals in Halle der Anempfehlung dieses Verfahrens keine vorurtheilslose Kritik entgegengebracht wurde.

Doch Angelegenheiten irgend einer Wissenschaft werden nicht durch die, Stimmung des Tages erledigt, und so bleibt es mir unbenommen, auf alle Einwände zurückzukommen. Ich habe abgewartet, um Thatsachen gegen Meinungen reden zu lassen, welch letztere nur Ausdruck der Tagesstimmung waren, sich aber nicht, oder doch nur theilweise, auf eigene Erfahrungen stuitzen konnten.

Es wurde diesem Verfahren Umständlichkeit vorgeworfen. Mit welcher Begründung kann dieser Vorwurf erhoben werden?

Dass das Durchstossen einer Spicknadel und das Abbinden des Stumpfes in zwei Partien rascher geht, gebe ich zu. Aber kommt es da auf einige Minuten mehr an, wenn andere wichtige Vortheile eingetauscht werden? Vortheile und Nachtheile dieses Verfahrens können überhaupt nur mit den intraperitonealen Methoden verglichen werden, und diesen gegenüber bietet es 1) die grösste Sicherheit gegen Blutung und gegen Blutnachsickern in die Bauchhöhle, 2) vollen Schutz gegen nachträgliche Infection des abgeschnürten Stumpfes von der Vagina her und die möglichste Sicherheit; dass, wenn je während der Operation eine Infection stattgefunden haben sollte, dies keine anderen Folgen als die Bildung eines abgeschlossenen Abscesses nach sich zieht. Gerade weil das Peritoneum nicht durchstochen und abgebunden, sondern über den abgeschnürten Stumpf gezogen und über demselben vernäht wird, kommt das Schnürstück nie völlig ausser Ernährung, und alle Vorgänge in diesem Gewebe sind von der Bauchhöble abgeschlossen.

Die anderen intraperitonealen Stielbehandlungsmethoden verwenden zur Blutstillung $\mathrm{Nähte,} \mathrm{und} \mathrm{davon} \mathrm{ist} \mathrm{das} \mathrm{Verfahren}$ 
von Schroeder mit der Etagennaht das verbreitetste. Wo wird die grössere Umständlichkeit liegen, bei etwa 3 bis 5 Partienligaturen und einigen sero-serösen Nähten oder bei 60 bis 200 muskulären Nähten in 4,5 und 6 Etagen über einander, wie dies Brennecke (a. a. O. S. 6) von seinen Operationen angiebt? Und trotz der zahlreichen Nähte, welche bei dickeren Stielen nothwendig sind, sichern dieselben nicht ebenso vollkommen gegen Nachblutung in die Bauchhöhle, als Ligaturen. Alle Nähte treffen die Gefässlumina mehr oder weniger in deren Achse, nicht quer. Ein Durchsickern von Blut und Serum ist eher möglich, als bei Ligaturen, und wie die oben erwähnten 2 Fälle wahrscheinlich machen, ist stets darauf zu rechnen, dass sich die Verhältnisse nach dem Erwachen aus der Narkose und durch die Contractionen und Relaxationen der verwundeten Uterusmuskulatur in Beziehung auf Blutungen weit ungünstiger gestalten, als während der Operation.

Je mehr Nähte ein Operateur legt, um so geringer wird diese Gefahr, und die überaus subtile Art der Nahtanlegung Brennecke's') erklärt dessen besonders gute Erfolge. Doch stehen meine eigenen Ergebnisse keineswegs denen Brennecke's nach.

Nun giebt dieser Forscher an ${ }^{2}$ ), dass ,er bei der Myomektomie durchschnittlich mit wenigstens zwei Stunden Dauer rechne", also gelegentlich noch mehr brauche. Das ist an sich bei 60 bis 200 Nahtstichen allein für die Versorgung des Uterusstumpfes kein Wunder, aber diese Erzählung bildet den Commentar für mein oben gebrauchtes Wort „Umständlichkeit". Dies ist der Nachtheil der Etagennaht, wenn sie ängstlich genau ausgeführt wird, und wo dies nicht der Fall ist, ist die Gefahr eine bedenklich grosse.

Es ist doch ein grosser Unterschied, ob man zu einer Myomektomie durchschnittlich wenigstens zwei Stunden braucht, oder mit einem Drittel dieser Zeit auskommt.

Darin irrt Brennecke, wenn er (a. a. O. S. 9) annimmt, dass, wenn ich jemals die Schröder'sche Methode selbst geübt und geprüft hätte, ich eine Abänderung nicht für nöthig gehalten hätte. Ich habe mir nie, auch nicht bei diesem Falle, ein Urtheil ohne eigene Prüfung erlaubt und von der Ausführung dieser Probe in meiner Monographie ausdrücklich und ausführlich geschrieben.

1) Zeitschrift für Geburtshülfe und Gynäkologie, Bd. XXI, S. 6.

2) Ebendas. S. 19. 
Hauptsächlish drehte sich die Verhandlung in Halle um die Infection des Schnürstumpfes vom Cervicalcanaleaus. Warum soll dies nun eher stattfinden bei der fortlaufenden Partienligatur, als der Etagennaht? Etwa weil ein bis zwei Catgutfäden mitten durch den ausgeschnittenen Cervicalcanal gehen? Ich widerspreche diesem Einwande entschieden. Erstens ist der Cervicalcanal von vornherein keimfrei, wenn man nicht sondirt hat.

Diesen Grundsatz kann ich aufstellen und festhalten nach Untersuchungen, die sofort nach der Exstirpation von Uterusmyomen mit Impfungen auf Nährgelatine ausgeführt wurden. Natürlich kommen Spontaninvasionen ausnahmsweise vor. Sieht man doch, dass submuköse Myome, welche, aus ihrer Kapsel getrieben, ausser Ernährung kamen, ohne irgend ein Zuthun in Fäulniss übergehen. An der Allgemeingültigkeit dieses Satzes, dass der gesunde Uterus keine Mikroorganismen in sich birgt, vermag die Mittheilung von Boisle $\mathrm{ux}^{1}$ ), dass er $4 \mathrm{Mal}$ unter 10 Fällen Mikroorganismen und davon 2 Mal pathogene Keime gefunden habe, nichts zu ändern.

Der Möglichkeit, dass Keime, selbst pathogene, in das Gebärmutterinnere vorgedrungen seien, muss auf alle Fälle Rechnung getragen werden, weil man im Augenblicke des Handelns nie weiss, ob im gegebenen Falle Immunität oder eine latente Invasion bestehe. In jedem Falle brenne ich nach dem Ausschneiden des Gebärmutterkörpers den Cervicalcanal mit dem ThermokauterFlachbrenner aus, so lange der provisorisch umgelegte Schlauch abschnürt, und schneide den Schorf noch tief heraus, um eine bessere Verheilung der Wände unterhalb der Schnürfurche zu erzielen. Warum soll hier eher eine Infection statthaben, als bei der Catgut-Circulärnaht, welche Olshausen zum Abschlusse des Cervicalcanales anlegt?

Schliesslich entscheidet über eine solche Frage der klinische Erfolg. Wenn ich hier eine ganze Reihe glatt, ohne Abscessbildung geheilter Fälle vorbringen kann, so ist denn doch für Unparteiische ein wichtiger Anhalt erbracht, dass es möglich ist, dem Gespenst der Infection vom Cervicalcanale aus zu wehren.

Ich wiederhole, dass diese Infectionsgefahr jede intraperitoneale Methode in sich birgt, auch die Schröder'sche, die, wenn sie bis zur vollen Blutstillung durchgeführt wird, auch

1) Bericht über die Verhandlungen des $X$. internationalen medicinischen Congresses, S. 6. 
Ischämie erzeugt, also ebenfalls für die pathogenen Mikroorganismen eine besondere Disposition schafft.

Um eine Eiterung im abgeschnürten Stumpfe zu vermeiden, ist die Hauptsache, dass derselbe nicht inficirt werde, aber selbst für den Fall einer Infection ist es sehr bedeutungsvoll, ob derselbe vollkommen oder nur vorübergehend aus der Ernährung geschaltet werde. Ich habe schon in meiner Monographie (S. 76) hervorgehoben, dass auf frische Operationswunden weit häufiger, als wir es annehmen, Entzündungserreger gelangen, aber im lebenden Gewebe unschädlich gemacht werden. Versenkt man dagegen einen abgebundenen, aus der Ernährung ausgeschalteten Uterus oder Myomstumpf, so wird damit ein Experimentum crucis ausgeführt, ob zufällig Keime auf den Stumpf gelangt seien oder nicht. Im ersteren Falle ist beim todten Gewebe auf ein Aufkeimen, also auf eine Eiterung zu rechnen. Wohl kann durch die Schnürfurchen oderwdurch Verlöthung des Peritoneum sich in dem abgeschnürten Stumpfe rasch eine nothdürftige Ernährung einrichten.

Der Widerspruch, den Fritsch ${ }^{1}$ ) u. A. dem Satze entgegenstellten, dass ausser Ernährung gesetztes Gewebe in Nekrose oder Nekrobiose übergehe, würde ja erst recht für die Anwendung von Ligaturen sprechen. Wenn dieser Satz grundsätzlich falsch, also das Gegentheil richtig wäre, so müsste ja die Massenligatur bei der Versenkung um so weniger bedenklich sein. Es ist jedoch die seiner Zeit in Halle $^{2}$ ) angegebene Begründung, dass man abgebundene Ovarialstiele noch ohne Gewebszerfall und Rückbildung gefunden habe, eine Erfahrung, welche völlig ungeeignet ist, die grundsätzliche Richtigkeit jenes Satzes zu widerlegen, weil in den abgebundenen dünnen Stielen eine zufällige anastomotische Ernäbrung in der Schnürfurche sich einstellen kann. Der Zerfall und die Gewebsrïckbildung sind bei den abgebundenen Geweben die Regel, die anastomotische Forternährung, auf welche sich meine Opponenten bezogen, die Ausnahme, auf welche nicht zu rechnen ist. Es war darum der eilige und schroff betonte Widerspruch gegen meinen Auspruch um so auffallender.

Es soll die Ernährung des abgebundenen Stumpfes gar nicht dem Zufalle überlassen bleiben, sondern durch Uebernähen mit

1) Verhandlungen der deutschen Gesellschaft für Gynäkologie in Halle 1888,

2) Ebendas. S. 153. 
dem Peritoneum der Collateralkreislauf von vornherein gesichert werden. Früher hatte ich das Peritoneum in der ganzen Breite mit abgebunden. Seitdem ich dies grundsätzlich vermeide, ist in einer langen Reihe von Fällen eine Eiterung oder Abscessbildung in der. Tiefe des Beckens und in der Umgebung des Stumpfes nicht mehr vorgekommen und derVerlauf stets so glatt gewesen wie nach Ovario-Kystomektomien.

Wenn Kaltenbach in der Verhandlung ') in Halle sagte, „dass die Methode der fortlaufenden Partienligatur eine Rïckkehr zu den früher gebräuchlichen Massenligaturen sei, deren geringerer Werth gegenüber Naht und einer mehr directen Unterbindung der Gefässbündel, wie bei der Methode von Schröder, doch allgemein anerkannt werden müsse", so kann dieser Ausspruch nur auf Missverständnissen beruhen.

Ich möchte in Entgegnung darauf hinweisen, dass ,eine mehr directe Unterbindung der Gefässbündel"“, wie bei der Methode von Schröder, doch hierbei ebenfalls stattfindet. Es beginnt die Operation mit einer Partienligatur der Ligamenta lata, wobei die sämmtlichen Arterien in ihren Stämmen unterbunden werden. Der geringere Werth, von dem Kaltenbach sprach, könnte sich also nur speciell auf die Behanḋlung des Uterusgewebes beziehen. Hier tritt jedoch ausschliesslich Naht gegen Ligatur in Vergleich; denn die directe Unterbindung der Arterienstämme geht hier, wie auch bei Schröder's Etagennaht, vorhèr. Eine Rückkehr zu früher. geübten Methoden ist sie, doch mit wesentlichen Unterschieden. Es scheint ja oft, als ob man sich im Kreise drehe.

Sicher ist dies bei dieser Behandlung so wenig der Fall, als bei der Versenkung der Ovarialstiele. Auch hier ist man zu alten Methoden zurückgegangen; hier war der. Nebenumstand, welcher das alte Verfahren zum erfolgreichen umgestaltete, die Antisepsis, bei der Myomektomie muss zur Bessergestaltung die Antisepsis und eine solche Art der Massenligaturen gefordert werden, dass man der Blutstillung sicher ist. Dann hat auch hier das alte Verfahren andere Erfolge, als früher. Diese beiden Punkte waren früher ver-

1) Verhandlungen der deutschen Gesellschaft für Gynäkologie 1888. II. S. 150 . 
nachlässigt. Uebrigens ist es ganz richtig, stets, wo es möglich ist, an die Rückkehr zum Alten zu denken! Denn in der Regel hat man mit dem einfachsten begonnen, und man braucht an dem Einfachen oft nur wenig zu ändern, so ist es das Beste.

Bei der Ovariotomie ist man auf die früher gebräuchlichen Methoden mit allseitig so befriedigendem Erfolge zurückgekehrt, dass heute kein Mensch mehr an ein anderes Verfahren denkt.

Der sachliche Einwand Kaltenbach's kann sich, wie oben betont, nur auf die Behandlung des Uterusgewebes beziehen; nur darauf kann die Bemerkung ,vom geringeren Werth“ bezogen werden.

Bei der Versorgung des Stumpfes kommen durch den Uterus mehrere Massenligaturen an Stelle von unvergleichlich zahlreicheren Nähten. Ligaturen sollen also weniger blutstillend sein, als Nähte!? Es klingt eine solche Behauptung unleugbar paradox und wird unter Chirurgen manches Schütteln des Kopfes erregen. Ich kann sie einfach nicht begreifen und nur annehmen, dass sie auf einem Missverständnisse über die Methode bestehe. Doch darf ich nicht unterlassen, mich dagegen zu wehren, weil, wie der Erfolg zeigt, ein solches Wort Schule macht, sowie es einem Anderen in den Kram passt. Brennecke ${ }^{1}$ ) citirt den Ausspruch Kalten bach's vom geringerem Werthe der Massenligaturen gegenüber Naht und einer mehr directen Unterbindung der Gefässbündel wörtlich, wo es ihm passt, mein Verfahren zu befehden, so unzutreffend dieser Einwand ist. Zur Blutstillung ist die Ligatur, bezw. Umstechung sicherer, als die Naht! So lautet es bei allen Chirurgen und nicht umgekehrt.

Gegenüber der Bemerkung von Kaltenbach²), dass er nie bei seinen Myomexstirpationen die Ligamenta lata so bequem nach den Seiten ausgespannt und entfaltbar fand, wie meine Operationsmethode voraussetze, frage ich, warum, aus welchen Gründen dies meine Methode voraussetze? Meine Zeichnungen stellen dies dar, um übersichtlicher zu sein. Aber selbstredend habe auch ich keinesfalls dieselben so ausgespannt gefunden, wie sie die schematischen Zeichnungen darstellen. Uebrigens hat doch die Abbindung der Ligamenta mit dem Principe der Stielbehandlung nichts zu thun.

1) a. a. O. S. 8.

2) a. a. O. S. 150 . 
Nicht nur so gut, sondern stets sicherer als mit allen anderen Mitteln lässt sich mit Hülfe der Partienligatur die Abbindung der Ligamenta ausführen, ob dieselben ausgespannt oder der Kante des Uterus straff anliegend seien. Und wenn einmal die wenig gebogene Schiebernadel nicht gut herumzuführen ist, so verschlägt es an der Sache nichts, sie durch eine Aneurysmanadel zu ersetzen.

Nicht nur bei straff an den Uteruskanten liegenden Tuben, sondern auch bei starren Verwachsungen der Uterusadnexe im Beckenboden und bei Verwachsungen des ganzen Uterus in Fällen, wo die extraperitoneale Stielbehandlung gar nicht durchgeführt werden könnte, liess sich die intraperitoneale, wenn auch mit vermehrten Schwierigkeiten, erfolgreich vollenden. Gerade darauf bezieht sich der oben ausgesprochene Satz, dass die intraperitoneale Stielbehandlung leistungsfähiger sei, als die extraperitoneale.

In zwei der hier mit aufgeführten Fälle waren die Uteri so unbeweglich, dass sich der Gebärmutterhals, welcher schliesslich zur Stielbildung benutzt werden musste, nicht bis zur Höhe des horizontalen Schambeinastes emporziehen liess. Und doch liessen sich diese Operationen erfolgreich vollenden.

Schwer und anstrengend ist es in einzelnen Fällen, die Bruns'sche Schiebernadel durch das Collum uteri zu stossen, wenn dasselbe sehr derb und straff ist. Ein anderes Mal und besonders in einen puerperalen Uterus dringt das Instrument so leicht ein, wie in Butter. Ich möchte darauf hinweisen, die Nadeln gut geschärft zu verwenden und nicht durch langes Kochen, Glühen oder trockene Hitze stumpf zu machen. Dass ich bei der Operation auf jeden Stichcanal achte, dass es öfters nöthig wurde, noch nachträgliche Umstechungen zu machen, theils weil einzelne Gefässe von den Ligaturen, wo sie das Peritoneum freilassen, nicht gefasst wurden, theils weil sie mitten durchstochen waren, ist nicht zu leugnen. Dann mussten solche Punkte durch Umstechungen besonders gegen Blutung gesichert werden. Dass Egon v. Braun einen Fall erlebte, wo die Bauchhöhle voller Blut war, spricht keineswegs gegen die Methode. Wenn man Alles sicher unterbunden und gut aufgepasst hat, so kann keine Blutung nachfolgen. Gerade darin ist die Unterbindung der Naht überlegen. Bei Nähten genügt eine kleine Unvorsichtigkeit, um Nachblutungen zu erleben, bei fortlaufenden Unterbindungen darf 
man wohl den Grad der Unrorsichtigkeit nicht mehr als klein bezeichnen.

Zu allen Unterbindungen des Uterusstumpfes benutze ich durch Hitze sterilisirtes Rohcatgut, für die sero-seröse Decknaht ganz schwach gehärtetes (10 Minuten lang in einer Lösung von $1: 10000$ ) und trocken sterilisirtes Chromsäure-Catgut. Es hat dies den Zweck, dass der Uterusstumpf nach dem Schmelzen des Catgut möglichst bald wieder in die Circulation einbezogen wird. An der peritonealen Naht nehme ich resistenteres Catgut, um hier auf längere Zeit des Abschlusses gegen die Bauchhöhle sicher zu sein.

Ueberblickt man die Wandlungen der Forscher über die Myomohysterektomie im Laufe der letzten Jahre, so kann man dabei recht interessante Beobachtungen machen.

Zuerst waren es Bardenheuer und A. Martin, welche an Stelle der Myomohysterektomie die Totalexstirpation des Uterus vorschlugen. Dieser Vorschlag galt keineswegs den kleineren Myomen, welche noch von der Scheide aus nach der gewöhnlichen Methode auszurotten gingen. Nein, in allen Fällen wurde zuerst per laparatomiam die Geschwulst und das Corpus uteri von oben her und dann das Collum entweder von der Bauchhöhle oder von der Scheide aus entfernt. Dieses Collum uteri scheint einen Stein des Anstosses zu geben, dass man so radikal damit verfährt. Warum diese riesige Vergrösserung des Eingriffes? Dass man die Gefahren und Nachtheile vom Collum her umgehen könne, beweisen denn doch meine Fälle zur Genüge. Warum sollte der Uterusstumpf ein Gebilde sein, an welchem die Grundsätze der erhaltenden Chirurgie so Schiffbruch leiden, dass das Beste wäre, den Stein des Anstosses wegzuräumen?

Die betreffenden Operateure verstehen den kleinen Cervixstumpf nicht zu behandeln, und darum muss der ganze Uterus mit Stumpf und Stiel ausgerottet werden.

$\mathrm{Zu}$ meiner grössten Ueberraschung schloss sich auf dem letzten internationalen medicinischen Congresse ${ }^{1}$ ) in Berlin auch Fritsch und seither Chrobak diesem Radikalismus an: Nicht die extra-, nicht die intraperitoneale Methode, sondern die Totalexstirpation werde das Verfahren der Zukunft sein.

Die Ueberraschung über den Meinungswechsel ist um so

1) Bericht des $X$. internationalen medicinischen Congresses. Centralblatt für Gynäkologie, Extrabeilage, S. 64. 
grösser und berechtigter, als Fritsch in Halle seine Methode der Einnähung des Stieles in der Bauchwunde warm empfohlen hatte. Es liegt in diesem grundsätzlichen Wechsel eine sehr beredte Selbstkritik.

Wenn der Zuwachs an Mühe bei der Ausführung gering wäre, so hielte ich diese Prophezeiung für wahrscheinlich. Da jedoch der Unterschied ein sehr bedeutender ist, so glaube ich viel eher, dass ein Rückschlag gegen dieses radikale Lösen des Knotens erfolge, und zwar, weil es auf andere Weise mit grösserer Sicherheit auf Erhaltung des Lebens geht. Gerade die Exstirpation des Collum uteri macht technisch weit mehr Schwierigkeiten, als die Entfernung des Myoms mit dem Corpus uteri. Diesen Einwand mache ich durchaus nicht einfach theoretisch, sondern aus hinlänglicher eigener Erfahrung mit dieser combinirten Totalexstirpation des Uterus.

Die Erfolge der beiden concurrirenden Operationen werden das entscheidende Wort sprechen und nach meiner Ueberzeugung für die intraperitoneale Stielbehandlung mit einer entsprechenden Ligirung des Stumpfes den Ausschlag geben.

Was die Indicationen betrifft, so habe ich mich über dieselben schon früher ') ausführlich ausgesprochen. Da die Myome häufiger in den späteren Jahren des Geschlechtslebens bedenkliche und lebensgefährliche Störungen machen, da sehr viele, ja die überwiegende Zahl der Frauen mit einer symptomatischen Behandlung bis in das Klimakterium gelangen, wo dann die lebensgefährlichen Symptome in der Regel schwinden, wird die Indication zur Myomektomie stets individuell verschieden sein. Ganz anders, als bei den Ovarialtumoren, hat hier die symptomatische Behandlung ein Recht. Aber die Kehrseite bleibt nicht aus. Weil man hoffen kann, ohne Operation vielleicht bis in die klimakterischen Jahre durchzuhelfen, kommen auch mehr hoffnungslose, ja vernachlässigte und verdorbene Fälle zur Myomektomie. Es ist noch eine Zeit des Hangens und Bangens über den Indicationen; durch diese wird aber umgekehrt auch wieder die Statistik der Operation verschlechtert. Ein bestimmt nachgewiesenes Wachsen der Geschwulst zwingt besonders in jüngeren Jahren unbedingt zur Operation. Nie sollte man bei jüngeren Frauen mit der Operation zögern, wenn der Uterustumor bis über den Nabel

1) Stielbehandlung u. s. w., S. 66. 
gewachsen ist; denn die Colossaltumoren, welche den Thorax aufwärts drängen, bedingen leichter einen Shok, um der Blutmenge willen, welche bei der Operation mit einem Schlage aus dem Kreislaufe ausfällt.

Auch Blutungen, welche die Kranke stark anämisch machen und sichtlich herunterbringen, verlangen die Myomektomie. Sicher bilden auch Schmerzen, welche die Kranke von sich aus zu dem Entschlusse bringen, eine vollberechtigende Indication.

Einer Indication sind wir unter unseren Fällen verhältnissmässig häufig begegnet, die von anderen Forschern selten erwähnt wird, nämlich Einklemmungserscheinungen seitens der Blase. Die Kranken litten an allen Graden der Excretionserschwerung: Zuerst häufiges Drängen zum Wasserlassen, dann mühsame und unvollkommene Entleerung, bis schliesslich die Unmöglichkeit der Harnausscheidung, also die Harnverhaltung mit all ihren schlimmen Folgen eintrat (Ischuria paradoxa). Wie bei der Retroflexio uteri gravidi und der Prostatahypertrophie gesellte sich auch hier immer eine Blasenentzündung hinzu.

Zu Einklemmungen im kleinen Becken können selbstverständlich nur kleinere Myome Anlass geben. Zwei Mal nahm ich deswegen Anstand, die Frauen zu operiren, und hoffte nach mühsamer Heilung der Blasenentzuindung durch eingelegte Pessarien einer erneuten Einklemmung vorzubeugen. Doch die Ringe hielten nicht, und die Kranken kamen durch Erneuerung der Einklemmung in Lebensgefahr. Sicher gehören die zwar verhältnissmässig seltenen Einklemmungserscheinungen zu den unbedingten Indicationen.

Intelligente Laien pflegen dem Rathe zur Operation die Frage entgegenzuhalten, was dann aus ihnen werde, wenn sie sich nicht operiren lassen? Auch wir Aerzte müssen diese Frage beim Entscheide über die Operationen stets vor Augen haben bei allen, auch bei solchen Kranken, welche uns nicht danach fragen. Die Antwort darauf giebt einen Maassstab zur Stellung der Indication, die Prognose der Operation den Ausschlag.

Natürlich kann nur der Arzt dies abwägen. Er muss die Entscheidung treffen, und unter gewöhnlichen Verhältnissen beeinflusst seine Entscheidung den Entschluss der Kranken vollständig. Diese Entscheidungen dürfen aber nur nach wahrheitsgetreuen Unterlagen getroffen und nur mit wahren Gründen den Kranken gegenüber vertreten werden. 
Wenn dagegen der Arzt in mysteriösen Worten von bevorstehenden oder doch möglichen Gefahren spricht, ist die Entscheidung den Kranken trügerisch gemacht. Es besteht in den weitesten Kreisen eine entsetzliche Angst vor „Krebs", und nicht mit Unrecht. Mit Andeutungen dieser Art kann man alle Kranken mürbe machen.

Solche Andeutungen sind bei Menschen, die wirklich an dieser Krankheit leiden, ausnahmsweise nicht zu umgehen, weil sie sonst zu gleichgültig bleiben. Etwas ganz Anderes und nach meinem Urtheile Verwerfliches ist es jedoch, solche Andeutungen von Krebs gegen Kranke zu machen, wo von Krebs nicht die Rede ist, wo der betreffende Arzt in derselben Stunde im Briefe an den Hausarzt die Diagnose auf eine völlig harmlose Krankheit stellt, wo er also mit Bewusstsein und Berechnung den Kranken etwas Unwahres sagt, um sie durch den Schrecken fügsam zu machen und unter das Messer zu bringen. Es ist durchaus nicht erlaubt, sondern gewissenlos gehandelt, wenn auch der Satz vor den Kranken scheinbar nur nebenhin gesprochen wird, ,es könnte sonst noch Krebs daraus werden". Es giebt viele Anlässe im Leben eines praktischen Arztes, wo er die wahre Meinung verschweigen muss, aber keinen, wo er in bewusster Weise, also ohne irgend welche Selbstüberzeugung, den Kranken eine falsche Diagnose stellen darf, um sie für eine Operation zu bestimmen. 